\title{
A Simulation Environment for Hierarchical Process Chains based on OMNeT++
}

Falko Bause, Peter Buchholz, Jan Kriege, Sebastian Vastag TU Dortmund, Informatik IV

D-44221 Dortmund, Germany

ffalko.bause, peter.buchholz,

jan.kriege, sebastian.vastag\}@udo.edu

Author prepared version of a paper published in

SIMULATION, Vol. 86, No. 5-6, 2010.

http://dx.doi.org/10.1177/0037549709104236 


\title{
A Simulation Environment for Hierarchical Process Chains based on OMNeT++*
}

\author{
Falko Bause, Peter Buchholz, Jan Kriege, Sebastian Vastag \\ TU Dortmund, Informatik IV \\ D-44221 Dortmund, Germany \\ \{falko.bause, peter.buchholz, jan.kriege, sebastian.vastag\}@udo.edu
}

\begin{abstract}
$O M N e T++$ is a discrete event simulation environment primarily designed for communication networks. In this paper we present an approach to enable $O M N e T++$ to simulate complex hierarchical process chains. Process chains are a common modeling paradigm in the logistics area for the analysis and optimization of large process chains and have been intensely used in many practical applications. Their evaluation is supported by the ProC/B toolset, a collection of software tools for modeling, analysis, validation and optimization of process chains. Here we describe how $O M N e T++$ has been integrated as a new simulation engine into the toolset. The integration has to get over some core problems to allow a smooth interaction between $O M N e T++$ and the other tools: In particular, the $O M N e T++$ model description of the logistics network should be kept manageable, it should reflect the entire model structure and non-standard performance figures, being relevant for an economic evaluation, should be ascertainable in order to satisfy the specific needs of the application area. The paper highlights the main steps of the automatic transformation of a hierarchical process chain model into a hierarchical model in $O M N e T++$. Furthermore, we show how the transformation has been validated and how detailed performance figures can be evaluated with $O M N e T++$.
\end{abstract}

\section{Introduction}

For the development and operation of contemporary networks in logistics, model based analysis and in particular the use of discrete event simulation is becoming an important factor to ensure that the networks meet the requirements concerning technical measures like delivery times or service levels and, on the other hand, are also cost effective. In the past different workflows in a logistics network have been specified with process chains as a poorly descriptive tool that does not allow one to derive simulation models from the description. This, however, implies that required simulation models have to be specified on

${ }^{*}$ This research was supported by the Deutsche Forschungsgemeinschaft as part of the Collaborative Research Center "Modeling of Large Logistics Networks"(559). 
their own without any formal relation to the process chain model. Of course, this approach has the disadvantage that different models have to be created for one system with all the known problems of additional modeling effort or inconsistencies between the models. Thus, the use of the entire process chain model as a base model for a detailed simulation model of a logistics network is highly recommendable.

To realize this approach partially informal process chain models have to be enhanced by formal information necessary to build a simulation model and adequate software tools for simulation have to be available. Of course, simulation of process chains is not a new idea [1], but a general approach which allows one to refine a high level process chain into a detailed simulation model and which can cope with the complexity and size of models of today's logistics networks is still missing. Available simulation tools for this purpose are either restricted prototypes [2] or extensions of business process modeling tools [3]. In both cases the capabilities of representing and analyzing more complex models are limited. Available simulation tools for manufacturing systems [4, 5] that have been developed for large systems lack basic features which are necessary to model logistics networks and general simulation frameworks are too low level such that an adequate modeling of complex process chain models requires too much effort.

In the past we developed a class of hierarchical process chain models which include the necessary information to map them onto discrete event simulation models [6]. The model class, denoted as ProC/B, is based on a hierarchical description where activities of a process chain are performed by some function unit which itself can be a complex process chain or some basic unit describing the consumption of space or time. The resulting models may include an arbitrary number of hierarchical levels which form an acyclic graph. Originally, ProC/B models have been mapped onto simulation models using the tool HIT [7] which has been developed in the mid eighties for modeling complex computer and communication systems. HIT perfectly supports the hierarchical structure of $\operatorname{Pro} C / B$ models and allows the analysis of results according to arbitrary paths through the hierarchy which is an important feature, in particular, if economic measures where cost drivers become important should be evaluated via simulation. However, the use of HIT also introduces some serious limitations. Since HIT generates a simulation model in the language SIMULA, a runtime environment for SIMULA has to be available to run a simulation. Unfortunately, the number of available SIMULA compilers is rather limited. Furthermore, HIT as a nearly 20 year old tool does not support several modern features of an object oriented simulation environment like animation or interfaces to software tools for post-processing of results or for the administration of models. For these reasons we decided to integrate a new simulation tool in our modeling environment and to support a mapping of ProC/B models onto the corresponding models.

An adequate simulation platform has to observe the following requirements:

- The full ProC/B model world has to be mapped to the simulation model.

- The hierarchy of the process chain models has to be adequately represented in the simulation model.

- Detailed measures that are definable in $\operatorname{Pro} C / B$ should be analyzable in the resulting simulation models.

- The simulation tool has to be driven by the ProC/B interface. 
- Simulation should be easily made interoperable with other tools of the ProC/B environment like the optimization tool OPEDo [8] or the trace analyzer Traviando [9].

- The simulation environment should be stable, should allow the definition and simulation of large models and it should support modern features of object oriented simulation.

- The simulation environment should be freely available for research according to some adequate open source license.

The last two points restricted the number of available tools significantly since most available open source simulation tools where not adequate to really simulate, in an efficient and error-free way, large models as they result from large logistics networks. After a more detailed look on the remaining tools, our choice was $O M N e T++[10,11]$, a simulation environment generating simulations in $\mathrm{C}++$. Although $O M N e T++$ had been developed and used for communication systems, it is well suited for the mapping of hierarchical process chain models and it also fulfills almost all of the above requirements. Nevertheless, the mapping of ProC/B models onto OMNeT++ is far from being trivial since complex hierarchies have to be transferred from one view into the other.

This paper introduces the combination of $P r o C / B$ and $O M N e T++$ to build a new and powerful simulation environment for process models of logistics networks. First steps of the work presented here have been developed in [12, 13]. In the following section we briefly present the ProC/B formalism, the corresponding toolset and the tool $O M N e T++$. Afterwards, in section 3 it is shown how the hierarchical structure of $P r o C / B$ is mapped onto a module structure of $O M N e T++$. Then we show how the behavior of $\mathrm{ProC} / \mathrm{B}$ processes is performed in $O M N e T++$. Section 5 is devoted to the validation of the mapping, followed by a first comparison of $O M N e T++$ and HIT by means of small examples. The paper ends with the conclusions.

\section{Basic Software Tools}

The approach we present in this paper uses $P r o C / B$ as input format, maps the models to $O M N e T++$, simulates the resulting model using $O M N e T++$ and maps the results back to ProC/B. In this section we briefly present the main features of the ProC/B approach including the available toolset and give afterwards a brief introduction into $O M N e T++$. For further details about both tools we refer to the literature $[6,11]$.

\subsection{Introduction to ProC/B}

Pro $C / B[6]$ is a process chain-based modeling approach which is used in the collaborative research center "Modeling of Large Logistics Networks" 559 (CRC 559;[14]) for modeling and performance evaluation of logistics networks. ProC/B accounts for the specifics of the application area by capturing the structure in form of function units (FUs) and the behavior by process chains (PCs). In ProC/B, FUs might offer services, which can be used by activities of process chains. Each service is again described by a process chain.

Figs. 1-3 present an example of a ProC/B model representing a simplified freight village. A freight village is a node of a logistics network which provides facilities for storing goods temporarily and transshipment between several type of carriers. 


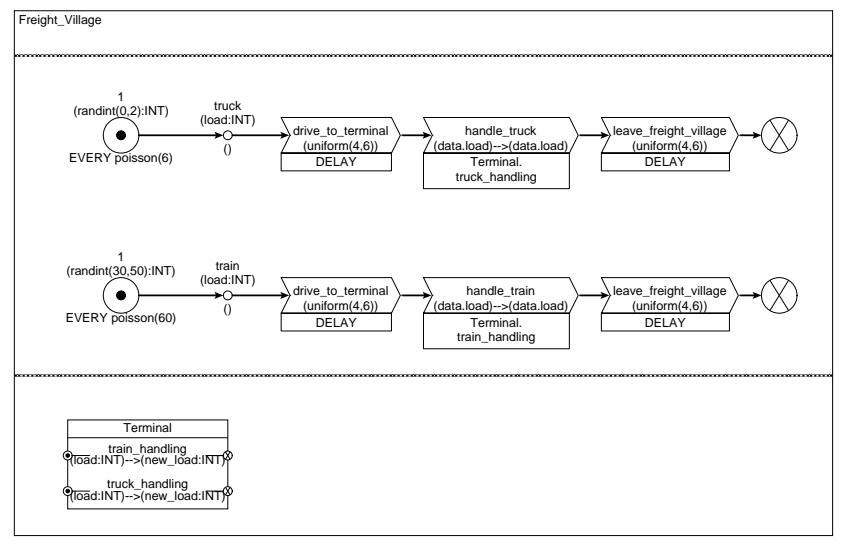

Figure 1: ProC/B Top level model "Freight Village"

The top level of the model (see Fig. 1) is specified by FU Freight_Village whose behavioral part is described by two PCs: truck and train. The structure part consists of a single (user defined) FU, named Terminal, which offers two services: truck_handling and train_handling. Services can be compared to functions in programming languages. In the example both services have an input parameter (load) and an output parameter (new_load). Behavior and structure part of a FU specification are interrelated by expressing which service of which FU performs an activity. In Fig. 1 the two PCs truck and train consist of three process chain elements (PCEs) each, and in both cases the second activity calls a service of FU Terminal. The inner view of FU Terminal is shown in Fig. 2. The offered services are specified by PCs and some of their activities use the services of two function units. One of them, FU storage, is a so-called standard function unit which offers predefined services (e.g. change). ProC/B offers two kinds of standard FUs: servers and storages. Servers (see e.g. pool1_forklifts in Fig. 3) capture the familiar behavior of traditional queues describing the consumption of time and storages describe the consumption of space (see storage in Fig. 2) and support the manipulation of passive resources. A simplified version of a storage is a so called counter, which is a standard FU often used for modeling synchronization aspects. A change to a counter or a storage is immediately granted iff the result respects specified upper and lower bound vectors; otherwise the requesting process gets blocked until the change becomes possible.

As indicated by the example ProC/B allows for the description of hierarchical models thus helping to cope with complexity. Fig. 4 shows the static structure of the ProC/B model of Figs. 1-3 exhibiting the relation of all FUs. User-defined FUs are displayed by squares and standard (pre-defined) FUs by circles.

Process chains directly visualize behavior. The freight village model of Figs. 1-3 reads as follows: Incarnations of process chain train are generated according to a Poisson distribution (with a mean of 60 time units). Each train has a load which is initially chosen by random according to an uniform distribution (between 30 and 50). After incarnation, the train "drives" to the terminal which is modeled here by a delay of the process for a uniformly distributed duration. Afterwards the train "is handled" by service train_handling of Terminal. This might result in a change of the train's load. Finally the train "leaves" the freight village and the process terminates at the sink. Considering Fig. 2 we see that handling a train means first to unload the train, which is possible if the storage's capacity of 300 units is not exceeded, otherwise the train has to wait until 


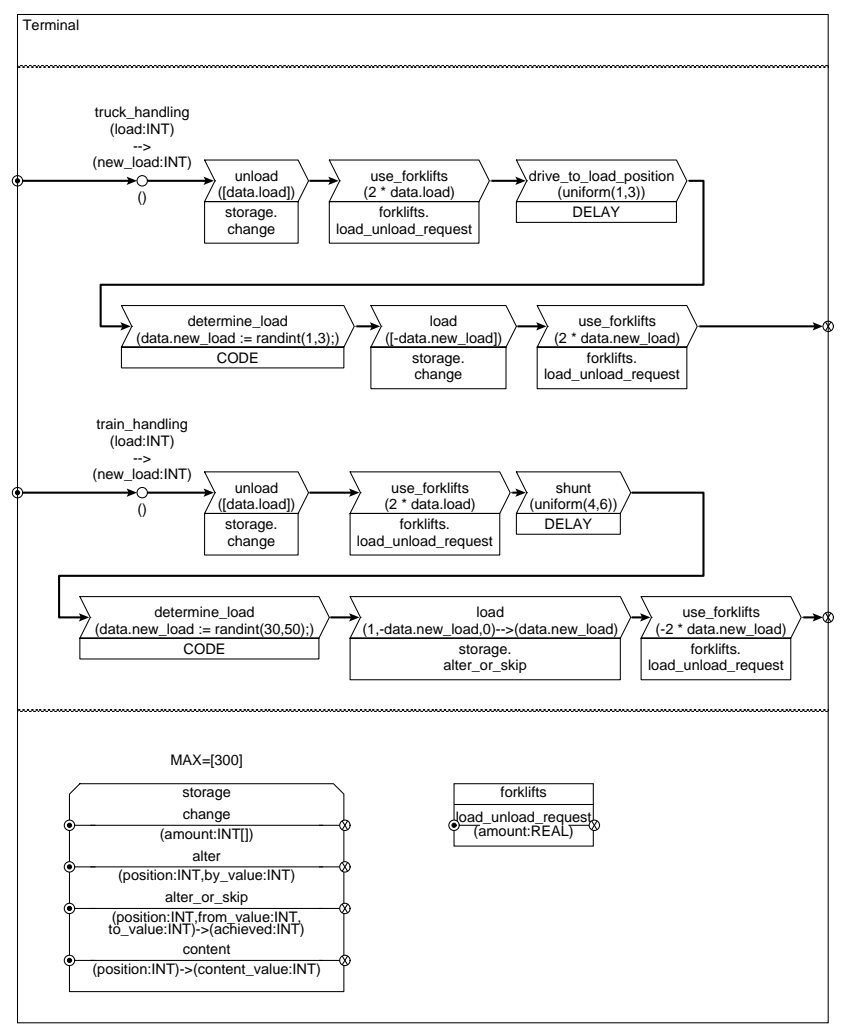

Figure 2: Function Unit “Terminal”

unloading is possible. Afterwards FU forklifts is called with the formal parameter amount of FU forklifts set to 2 * data. load". Afterwards the train "shunts" to a new position (which is again modeled by a delay of the process) and determines the new load. The new load is removed from the storage if possible, otherwise the available number of units are removed from the storage (which is the semantics of service alter_or_skip). Finally service load_unload_request of FU forklifts is called again before the process "leaves" the terminal. The behavior specification of FU forklifts is a bit more complex (cf. Fig. 3). After calling service load_unload_request a train equiprobably selects one of two pools of forklifts for service. This choice is modeled by a probabilistic OR-connector specifying alternative behaviors by different branches. After selection of a branch a train applies for service by incrementing one of the global variables req1 or req2 and waits for the availability of a worker who is needed to operate a forklift. Waiting is modeled by a so-called process chain connector which synchronizes processes of different process chains. In the example a train can only proceed to PCE use_pool1 or use_pool2 if a worker has arrived or is waiting at the corresponding process chain connector. After synchronization the train calls service request of the standard FU pool1_forklifts or pool2_forklifts resp. and parallel to this activity the worker decrements the variable req1 or req2 resp. in that way recording service of the train. In the shown model we do not distinguish between individual workers, and thus the worker waits for the end of the service of some train, which is again modeled by a process chain connector. Once the train has finished service at FU

\footnotetext{
${ }^{1}$ Access notations to parameters and variables of processes are prefixed with keyword data for technical reasons in order to distinguish them from global variables of an FU. Fig. 3, e.g., shows two global variables: req1 and req2.
} 


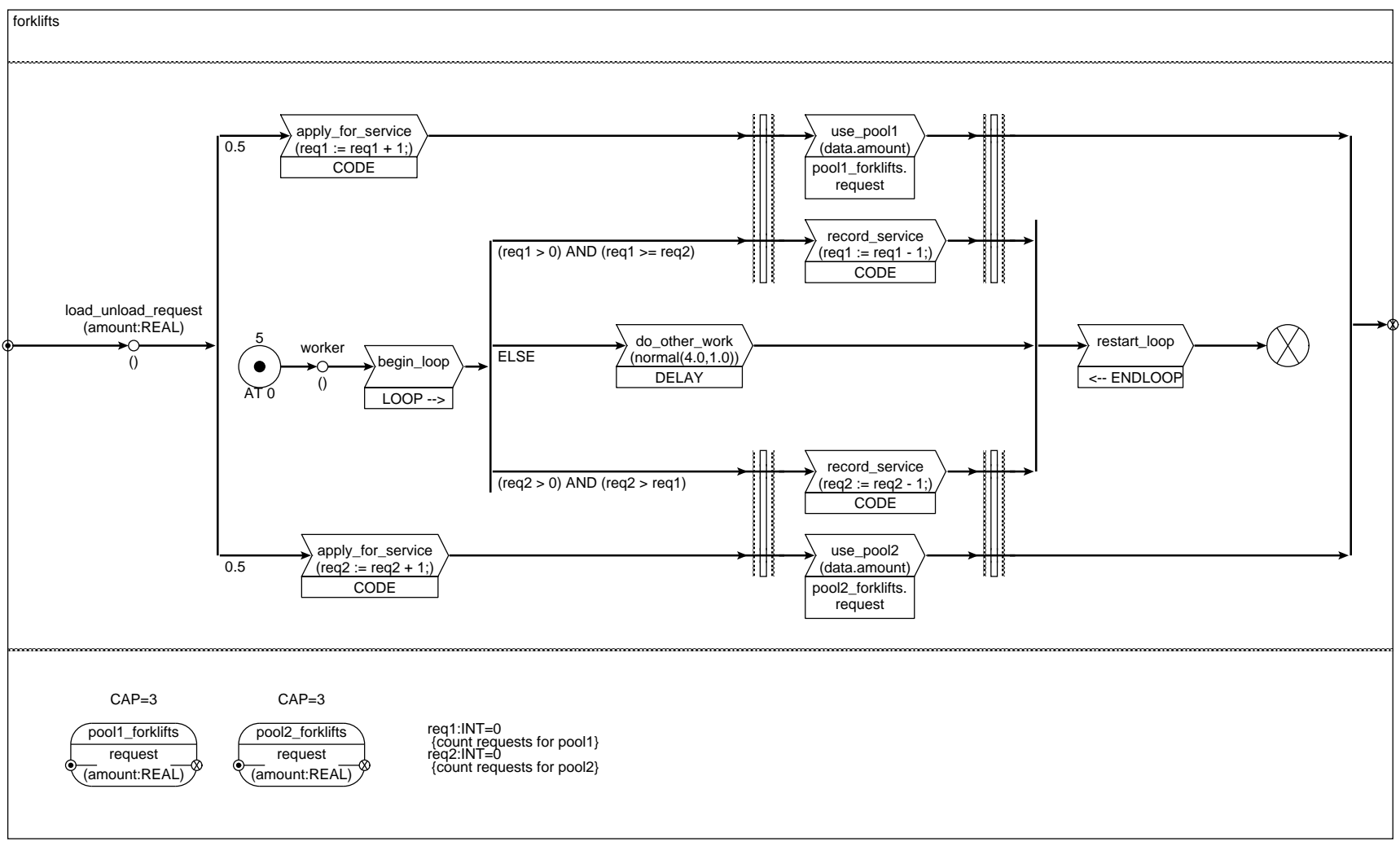

Figure 3: Function Unit "forklifts"

pool1_forklifts or pool2_forklifts it leaves FU forklifts. The behavior of workers within FU forklifts is modeled by PC worker. The source generates 5 workers at time 0 and each worker infinitely often repeats the behavior pattern described between the two loop constructs. The behavior pattern implements a simple control logic by 3 different branches which are selected according to a so-called boolean OR-connector. E.g., if trains (or trucks) have applied for service at pool1_forklifts by incrementing variable req1 the upper branch is selected by a worker provided the number of requests for pool1 is at least as much as for pool2. If no trains (or trucks) are requesting service (i.e. req1= 0 and req $2=0$ ) a worker is engaged in some other work which is modeled by a delay of the process.

The behavior of trucks and corresponding service calls reads similar to the described behavior of trains. In the sequel we will use the term process for the process description and its incarnations.

\subsection{Software Support for ProC/B}

In the course of the CRC 559 a toolset has been developed which provides a graphical user interface to specify $P$ roC/B models and transformer modules which map Pro $C / B$ models to the input languages of existing tools, so that Pro $C / B$ models can be analyzed automatically (cf. [6] and Fig. 5). Since modeling and analysis is intended to be used during the whole development cycle and also during the operation of a system, the toolset contains apart from modules to specify and simulate 


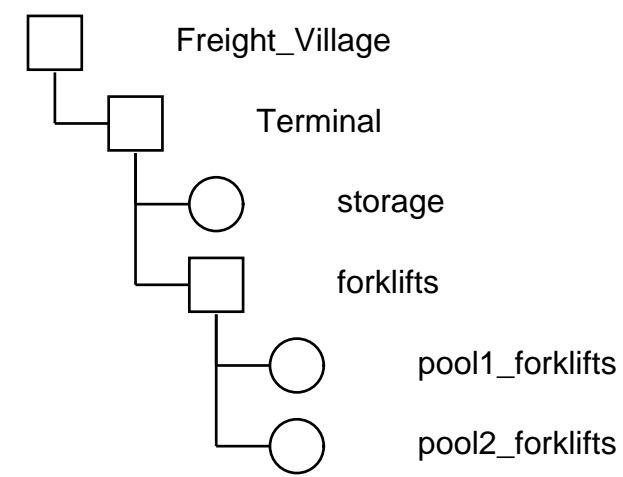

Figure 4: Hierarchy of model "Freight Village"

ProC/B models also modules for several non simulative analysis steps and for optimization. The ProC/B toolset has been successfully applied to different areas of logistics networks, such as for the modeling and analysis of freight villages, air cargo centers and supply chains [15]. We give a brief overview of the different parts of the toolset (cf. Fig. 5) with a particular focus on simulation.

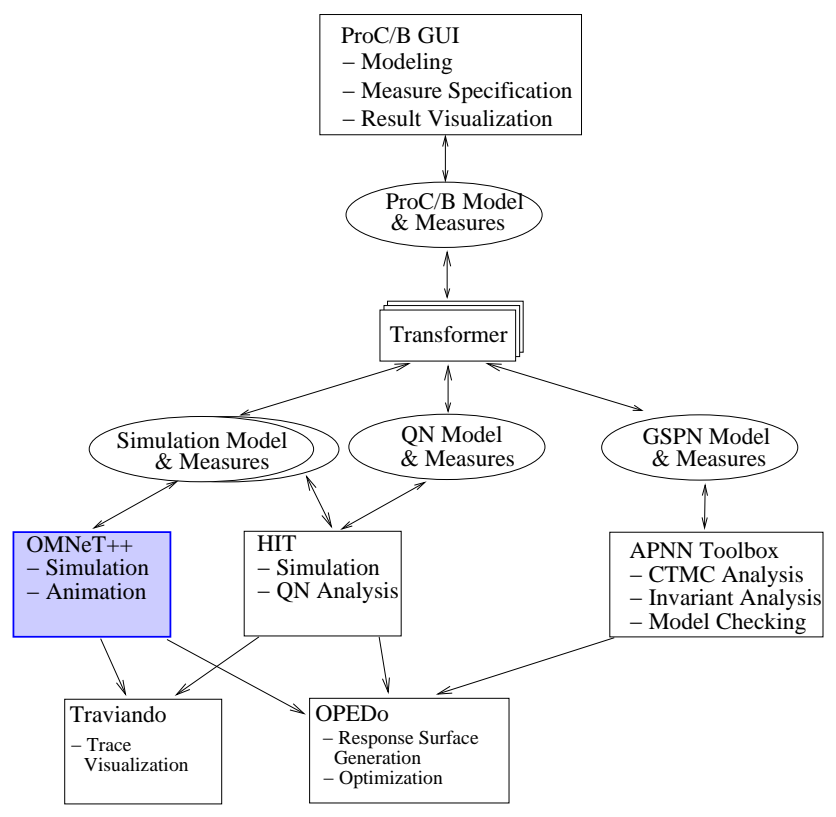

Figure 5: ProC/B toolset

\subsubsection{Graphical User Interface}

The graphical user interface (GUI) which resides on top of the whole toolset (cf. Fig. 5) allows the user to specify ProC/B models hierarchically by using a separate window for every non-standard functional unit. Standard functional units are described by their parameters. The hierarchical structure of ProC/B models supports the specification of huge and complex 
process chains in a user friendly way by considering only a small portion of the model in a single window. Additionally, the hierarchical and modular structure supports the reuse of modules by using standardized processes in logistics networks. Apart from the model, the user can specify measures in experiment descriptions. Experiments belong to a model specification and allow the definition of detailed measurements describing technical results like throughputs, sojourn times or utilizations as well as economical measures related to costs. ProC/B models and experiments are stored in a proprietary intermediate format which needs to be transformed to be used as input format for other tools. Currently, an alternative format for ProC/B models based on the standardized process description language BPEL [16] is under development [17].

From the interface different tools and operations can be started. It is possible to invoke the simulation and the transformation of ProC/B models into queueing networks or generalized stochastic Petri nets as briefly outlined below. Furthermore, one can use tools for result visualization and trace analysis. ProC/B models may as well be used as black box functions for optimization. The tool OPEDo [8] provides several different optimization techniques also being able to deal with stochastic functions.

\subsubsection{Non Simulative Analysis}

Simulation is the major analysis technique for ProC/B models, but also other techniques can be applied for analysis. Very prominent are queueing network analysis approaches which allow the very efficient analytical computation of performance measures like mean throughputs or sojourn times. Usually, QN analysis is applied in the early design stages when abstract models are used to obtain rough estimates for performance measures. In order to apply these analysis techniques, Pro $C / B$ models have to be mapped onto queueing networks which may not contain process synchronizations, simultaneous resource possession or general probability distributions. Thus, only ProC/B models without elements like storages, code elements and synchronization constructs can be used as an input for QN analysis. A transformer module maps the ProC/B description to the input language of a tool for QN analysis (cf. Fig. 5). After the QN analysis has been performed, results are mapped back to $P r o C / B$ and can be interpreted at the level of the process chain.

Another application area for non simulative techniques is the validation of simulation models. Complex simulation models, like any other complex computer program, may contain different kind of errors or bugs. Even if the hierarchical and modular description of $\operatorname{ProC} / B$ supports the specification of structured models, a user may still specify undesired or even wrong behavior. There are two classes of validation techniques, namely static techniques which use formal techniques to analyze the model structure and operational techniques which debug in some sense the output of the simulator. We briefly outline the use of static techniques here and introduce operational techniques in the following paragraph in conjunction with simulation.

Different approaches exist to validate simulation models during the whole life cycle [18]. In the ProC/B toolset Petri net (PN) techniques [19] are used to analyze functional and non functional properties. Classical PN techniques allow one to analyze properties like deadlocks or liveness of a system and recently we developed an additional PN-based technique to detect non-ergodic models [20]. All these, usually undesired properties of a model are often difficult to detect with simulation.

In order to apply PN techniques ProC/B models are mapped onto colored Petri nets with finite color domains. This class of Petri nets can be efficiently analyzed but cannot describe all behaviors of ProC/B models. In particular data dependencies 
are often difficult to describe or cannot even be modeled in a PN and thus are substituted by non deterministic choices. This implies that the PN includes some behaviors that cannot be observed in the simulation model and the user has to decide whether an undesired behavior in the PN is a valid behavior for the simulation model. Despite of this need for user support, PN techniques are a powerful tool to detect specification errors in ProC/B models [21].

\subsubsection{Simulation Techniques}

Simulation is the basic analysis approach applicable to all $\mathrm{ProC} / \mathrm{B}$ models. In the past, simulation was only supported by HIT [7, 22]. HIT is a modeling environment which does not only provide a simulator, but also offers efficient non-simulative analysis algorithms being based on product-form QNs and is also used as a QN solver in the toolset. HIT is basically tailored to steady-state analysis, based on a single replication approach. Time Series Analysis techniques are applied to individual streams of data produced by the simulation. A key feature of $H I T$ and thus of $P r o C / B$ is that these streams may be itemized in detailed ways. E.g., in Fig. 2 it might be of interest to measure separately the number of service calls for FU storage caused by trucks and trains. HIT provides facilities to describe and to evaluate measures for such activities at a lower level which are caused by some higher level originator and to itemize corresponding results with respect to the originators. As mentioned, $H I T$ is nearly 20 years old and needs a SIMULA compiler for execution. Therefore we recently integrated $O M N e T++$ into the $P r o C / B$ toolset trying to benefit from the features of a modern object-oriented simulation environment. In both cases, the ProC/B model is transformed into the specification language of the used simulation tool, the simulation run is performed and results are mapped back to ProC/B. The mapping onto $O M N e T++$ is introduced in the following sections after a brief overview of $O M N e T++$ has been given in the next subsection.

Simulation results can be presented in different forms. The simplest way is to present the raw numbers including confidence intervals in the graphical interface in conjunction with the corresponding model elements. Additionally, series of results can be defined to plot curves, e.g. to represent the evaluation of a measure over time. Apart from result measures, simulations can be used to generate detailed traces of the model behavior. Such traces are the base of animations and they can also be used to validate the simulation model or the system. The ProC/B toolset contains a specific tool Traviando [9] to represent and evaluate traces. Based on the idea of message sequence charts in UML, simulation traces of process oriented models are presented graphically and can be analyzed using different analysis approaches for functional analysis to detect properties like repetitive behavior, overloaded resources or unused communication relations between processes. These features help to validate a model and to get a better understanding of possible sequences of activities. As an example Fig. 6 shows the visualization of a trace generated by the model of the freight village. To keep the presentation compact only a small trace is used here, which contains the activities of a truck between its arrival at the freight village and its departure.

\subsection{OMNeT++}

$O M N e T++$ is a public-source simulation environment that has been developed for the modeling of communication protocols and has been extensively used in this area. Although it is mentioned on the web page [11] that OMNeT++ has been used for the analysis of business processes there is nothing available about this application and it does not seem that a complete 


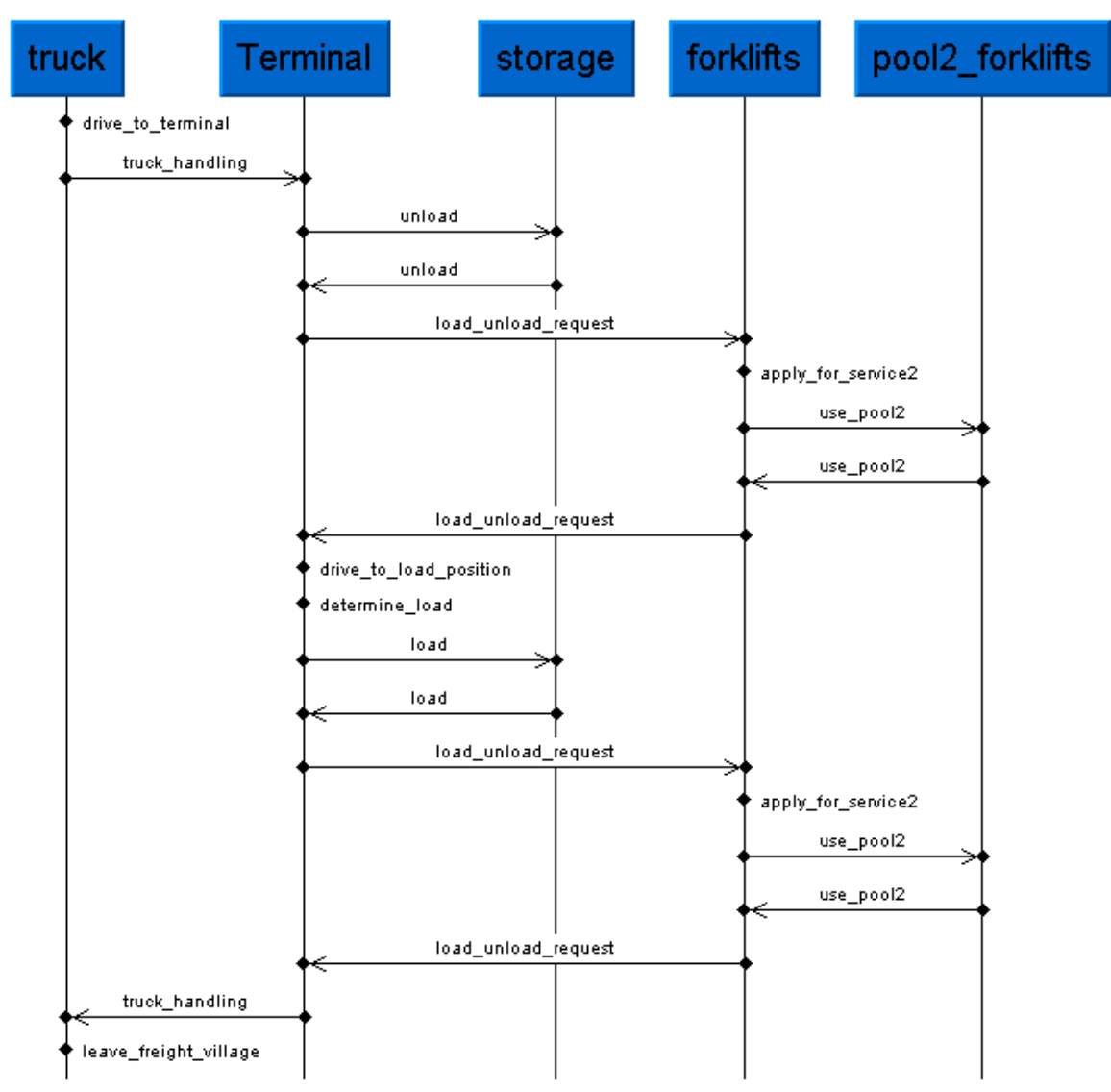

Figure 6: Sequence chart of a trace

mapping of hierarchical business processes onto OMNeT++ models has been done before. The whole tool environment includes a graphical front end and several other tools that support the modeling and simulative analysis of complex systems. Of particular interest is the simulation kernel which is written in $\mathrm{C}++$ and offers several classes to support the specification of complex hierarchical models. Furthermore, the resulting simulation models are known to be rather efficient.

The basic entities of an $O M N e T++$ simulation are modules. Modules can be simple, which means that they are implemented as C++ classes, or compound modules which implies that they are composed of other simple or compound modules. In this way OMNeT++ models are hierarchical. The complete model containing the overall hierarchy is denoted as the system module. Modules communicate via gates using messages. Gates can be input or output and a module may have an arbitrary number of gates. Messages are sent either directly to a gate or along a path. Basically paths are used to describe the transfer of messages over some medium. Therefore they offer parameters to specify e.g. the bandwidth or loss rate. The connection of modules via paths is specified in the *.ned file which includes the structure of the model and can be defined with the help of a graphical interface. The graphical interface can also be used for the animation of the running model by visualizing messages that are sent along a path from one module to another. In this way, an $O M N e T++$ model consists of two parts, the module descriptions in $\mathrm{C}++$ and the ned descriptions which specify the model structure given by the connection of 
modules.

An arriving message is interpreted in a module as an event and the user has to specify a routine handleMessage() for each arriving message type. Messages themselves can be structured data types and may include information that is used in the corresponding handleMessage() routine. In the routines new messages may be generated immediately or after some delay and already scheduled messages may be deleted. Thus, the basic event driven approach is realized by the processing and sending of messages. Apart from this general mechanism, the simulation kernel of OMNeT++ offers a lot of support to realize complex simulation models like up to date random number generators, support for statistical evaluation of results or support for parallel replications.

From this very brief description it should become clear that both, $P r o C / B$ and $O M N e T++$ use a hierarchical structure to describe models. However, at a second view it becomes clear that the model views differ in several yet important details. $O M N e T++$ has been designed with communication systems in mind such that messages have a physical meaning whereas in $P r o C / B$ hierarchy is introduced by calling services of FUs without explicit messaging. Another important aspect is, as already mentioned, the definition of detailed and origin dependent measures which are not directly supported by $O M N e T++$ and therefore have to be implemented separately.

The challenge is to get a correct mapping from ProC/B onto OMNeT++. Since correctness of the mapping cannot be formally defined because only a subset of $P r o C / B$ has a formal semantics in form of a Petri-net mapping [21], we define correctness by comparing the simulation using HIT and OMNeT++. The HIT simulation is usually taken as the correct behavior, since we defined an operational semantics of the whole ProC/B paradigm via HIT [23], and thus the OMNeT++ model has to show the same behavior. Of course, a detailed comparison implies that the model is completely deterministic since otherwise different random number streams will necessarily result in different behaviors such that only statistical results can be compared using adequate statistical methods [24]. We used both, simple deterministic models to show that the basic behavior is the same and more complex stochastic models to compare statistically the result measures.

Of course, if $O M N e T++$ is used as simulation kernel for ProC/B models, then one may as well use $O M N e T++$ modules in $P r o C / B$ models by including them in code elements. In this way it is possible to realize communication in process chains in a very detailed way. E.g., two different processes described in $P r o C / B$ communicate via a computer network specified in $O M N e T++$ using predefined protocol models. In [25] this approach is presented to model service oriented architectures where the services are described by $P r o C / B$ models and communication among the Internet is realized in an $O M N e T++$ model. Thus, the presented mapping of $P r o C / B$ onto $O M N e T++$ enables the definition of very large heterogeneous simulation models. However, the completely automatic specification, simulation and evaluation of those models is still a subject of ongoing research.

In the following two section we first describe how the hierarchical structure of a $\mathrm{Pro} C / \mathrm{B}$ model is mapped onto a corresponding structure of an $O M N e T++$ model. Then the mapping of the behavior is presented. Both steps are accompanied by small examples showing the basic ideas. 


\section{Mapping of Structure}

Pro $C / B$ models are specified graphically in the Pro $C / B$ editor and are stored in files. Next to the model itself the editor allows for saving experiment descriptions in separate files. As stated above, these general model/experiment description files can be used in different analyzers, either numeric or simulative. Thus, the generic model descriptions generated by the editor have to be translated to specific input formats.

Our implementation of mapping ProC/B to OMNeT++ consists of two main components: The converter procb2ned and a library named Osimu containing generic implementations of ProC/B's behavior as simple modules for $O M N e T++$. OM$\mathrm{NeT+}+$ requires behavior to be located in simple modules, that are written in $\mathrm{C}++$ and handle arriving messages in order to trigger specific reactions. The $\mathrm{C}++$ sources of simple modules are combined with *.ned-files using an identical naming scheme, describing module interfaces to $O M N e T++$ 's simulation system. The Osimu library contains a predefined simple module for each type of element of a ProC/B-model, except user defined FUs, capturing the behavior of those elements. These simple modules, like sources, PCEs, OR-connectors, process chain connectors etc. are configured by parameters specified in the corresponding *.ned-files. The library will be treated in Sect. 4 in a more detailed manner.

The converter procb2ned reads process chain models and outputs $O M N e T++$ network descriptions (*.ned-files) as a direct input format for the $O M N e T++$ simulation system. As $O M N e T++$ supports hierarchical modeling of modules these *.nedfiles describe the hierarchy of the model as compound modules. In ProC/B-models the hierarchy is represented by user defined FUs usually including at least one process chain offered as a service by the FU. procb2ned creates a *.ned-file, i.e. a compound module, for every FU preserving the structure of the ProC/B-model. As already mentioned for every language element in ProC/B exists a corresponding implementation as a basic module in $O M N e T++$. For model design these basic modules are instantiated and related by connections in a *.ned file, forming a compound module. Non-basic modules can be used similar to basic modules, making it easy to build hierarchical models. PCEs only form linear structures at the same model level, so procb2ned simply inserts them as basic modules into its output *.ned files. Of course, synchronization and event driven generation of new processes are also possible in ProC/B using available language elements (see [6]) which are realized as $\mathrm{C}++-$ implementations in $\mathrm{OMNeT++}$. Following the rule of one module per ProC/B language element, StandardFUs like ServerFU, StorageFU and CounterFU are also inserted directly into the model. If the converter reads a constructed FU on input, it goes one level down in the recursion, applying the above mapping rules to a new *.ned file named after its FU in $P r o C / B$. After returning from recursion, the compound module representing a constructed function unit can be used like any basic module. The subsequent step is to map process flow through a process chain by establishing connections between modules. The acting entities of $P r o C / B$ are all processes within a module. While processes are no specific object-types in HIT, it was a natural choice to map exactly one process type to exactly one message type in $O M N e T++$. Hence, ProC/B's connections between process chain elements are mapped to module connections in $O M N e T++$.

As shown in Fig. 1, connections in ProC/B only exist within a PC specification, there are no explicit connections from PCEs to FUs or the other way round. Only implicit relations between PCEs and FUs exist by specifying parameters in PCEs denoting which FU and offered service they call.

We transferred this idea to $O M N e T++$ by using traditional message passing through gateways and direct message sending 
as two separate forms of connections. The first task is done straightforwardly by procb2ned. Basic modules act as PCEs (excluding sinks) and obtain one connection to their successor, forming a structure similar to process chains in ProC/B. For this purpose, every PCE module has at least one set of input/output gateways acting as a socket for OMNeT++'s connections.

Again, relationships between modules of PCEs and FUs exist only implicitly in OMNeT++. Two parameters are given in the *.ned file for every instance of a PCE using a function unit: The identifier of the FU and the name of the offered service (keeping in mind that FUs can offer multiple services). This information is used in the PCE initialization phase to find the reference to their loosely bound function unit. Requesting a service in the simulation phase is done by transferring messages directly using OMNeT++'s sendDirect ( ) method to the function unit bound to the service. Finishing a FU's service is also signalized by returning the message.

Using OMNeT++'s alternative way to transfer messages has some advantages compared to the traditional way of using module connections:

1. As FUs can be used by possibly infinitely many PCEs, omitting explicit connections helps to keep models concise. The target to which direct messages are sent to is determined during the initialization phase by $O M N e T++$ and saved as a reference, so no extra time is consumed when analyzing the model.

2. Messages sent directly keep track of their senders on a stack, so returning a process message to its sender after performing a service is a simple task in the FU's implementation.

3. The visual appearance in the ProC/B editor and $O M N e T++/$ Kenv is kept similar.

Function units need one input gate per offered service. Output gates are redundant here, as the virtual sink terminating the service's process chain will return the message via direct transfer to the calling PCE. The ability to send and receive direct messages requires some preconditions for modules in $O M N e T++$ : Direct messages can only be delivered to dedicated input gates without any other incoming connection. Therefore, PCE modules calling FUs need an additional input gate reserved for callbacks of their associated FUs. By convention, new processes arrive at the first, status messages from FUs at the second input gateway.

An example of the output generated by procb2ned can be found in Listing $1^{2}$. The Listing contains an excerpt of the compound module generated for the top level FU of Fig. 1 containing the simple modules corresponding to a source, a Delay-PCE and a PCE calling a service of the contained FU Terminal. The simple modules are configured by parameters, e.g. describing the interarrival times for the source or the delay of the PCE. More information about simple modules can be found in Sect. 4. Furthermore, the *.ned-file contains a section specifying the connections between these simple modules.

\subsection{Animation}

An important additional benefit of using $O M N e T++$ for simulating ProC/B models is the animation capability of $O M N e T++$ 's graphical workbench $O M N e T++/$ Tkenv. Existing ProC/B analyzers are tuned according to efficiency and performance of the solution and are consequently batch processing systems, making it difficult to explain the dynamic behavior of processes

\footnotetext{
${ }^{2}$ For a unique naming scheme $\operatorname{Pro} C / B$ variables and names are prefixed.
} 


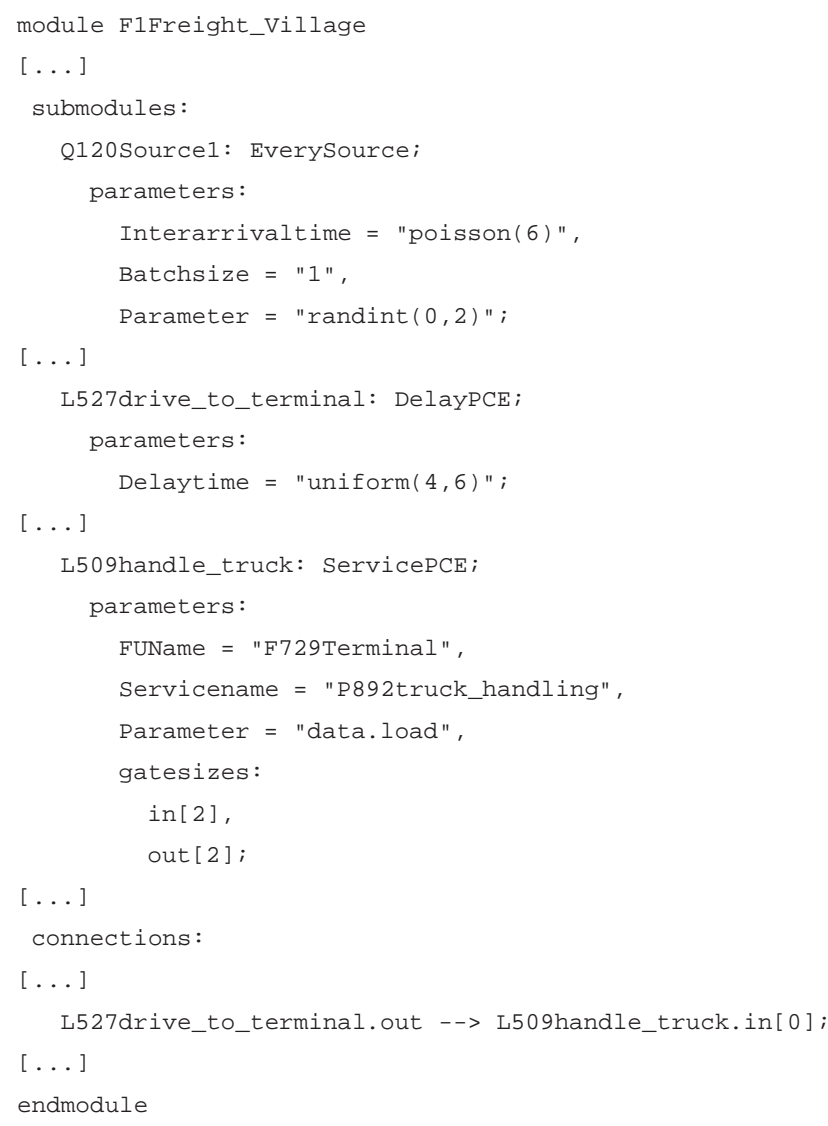


from the model. However, such an explanation is often important in teaching and also in real projects as we noticed when modeling large systems in cooperation with real users. Using $O M N e T++/ T k e n v$, messages moving between modules can be animated by a moving red dot as an adequate visualization for processes moving through process chains and making use of FUs. In this way, the dynamics of a system is clearly visible.

Pro $C / B$ 's graphical representation was carried to $O M N e T++$, using $O M N e T++$ 's feature to define pictograms for modules. procb2ned assigns bitmaps to every instance of the basic module matching its type in $P r o C / B$.
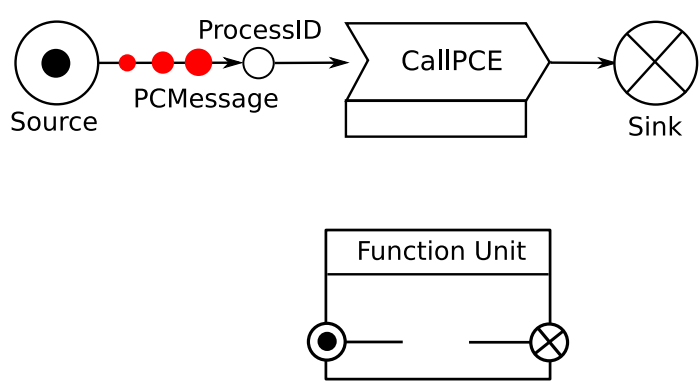

Figure 7: Animation of PCMessages

When setting $O M N e T++$ to a "slow" running mode in $O M N e T++/ T k e n v$, the user can trace processes created by a source as a red dot moving along the process chain element's outgoing connection (see Fig. 7). Arriving at a PCE, the dot is delayed until the PCE ends its call to an FU. Since no permanent connection exists between PCEs and FUs, a temporary connection is drawn acting as a path for messages performing a request by being sent directly to the module of the FU (Fig. 8). When the request is served, a message can be seen moving backward to the calling PCE on a reverse connection. Furthermore, different windows can be opened to view the animation simultaneously at different levels, i.e. in different FUs.

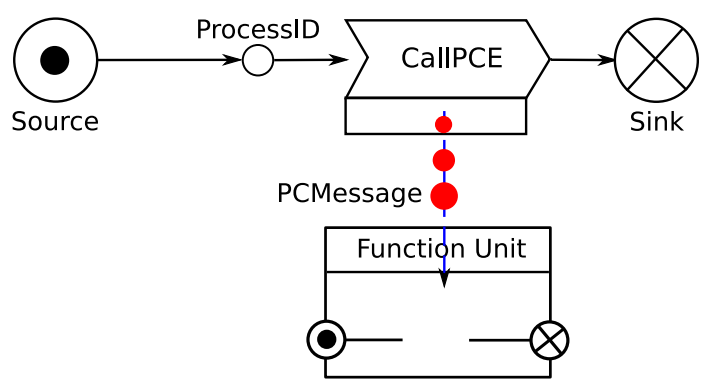

Figure 8: PCMessage moving over temporary connection

\section{Mapping of Behavior}

The second component of our framework is the mapping of the behavior of $P r o C / B$ language elements onto modules in $O M N e T++$. These modules are completely configurable by passing parameters, so we were able to compile a library $O$ simu 
containing generic implementations for PCEs and standard FUs.

$O M N e T++$ offers two different programming styles, lightweight processes and a transaction based programming paradigm. We decided to follow the latter as it matches the basic ideas of process chain models and it scales much better for large models. Since process chains are characterized by the interval of time consumed by a process between entering and leaving a chain, a transaction based discrete event approach makes it natural to map these intervals to arrivals and departures of messages to/from modules in $O M N e T++$.

The dynamic part of $P r o C / B$ models are processes following the route defined by process chains. Analogously, processes are represented by messages in $O M N e T++$. The mapping to $O M N e T++$ is done by subclassing cMessage only once to PCMessage (short for "process chain message"). Processes are marked with a unique identification number to trace their movement inside the model for debugging and statistics. In ProC/B, transitions of processes between PCEs are instantaneous, time is consumed by requesting services at function units or by dedicated delay PCEs. This idea is reproduced in $O M N e T++$ by messages of type PCMessage that use connections in zero time, leaving progress of model time to the modules.

Implementation of ProC/B's behavior is completely located in basic OMNeT++ modules, the leaves of the model tree. Compound modules used in $O M N e T++$ to group simple modules to complex model elements are not allowed to define own behavior in $\mathrm{C}++$ code next to their *.ned-file. For this reason the behavior of higher level FUs had to be the union of semantics defined by lower level PCEs, FUs and the way they are connected with each other. In syntactic terms we had to flatten the model hierarchy to basic $P r o C / B$ elements and connections.

This is an important difference to the former way $P r o C / B$ was implemented. Several functions regarding time progress, message routing and measurement are located in every node of the model tree of HIT. In the following we will describe our solution for the implementation of ProC/B's behavior with $O M N e T++$. Section 4.1 will also focus on an alternative to add measurements to specific constructed FUs.

The ProC/B-mapping to $O M N e T++$ is conducted with an inheritance hierarchy. Each implementation of a basic ProC/B element inherits from ProCBElement. ProCBElement itself is a direct successor of OMNeT++'s modeling interface CSimpleModule. ProCBElement encapsulates several common functions for ProC/B elements. On initialization, each ancestor of ProCBElement will register itself to the system. This allows faster location of ProC/B elements by name or by their position in the model hierarchy. Sending process messages direct or via connection is also implemented at this place. Keeping such basic functions at a high level in the inheritance hierarchy helps to keep a model wide consistent numbering of input and output gates for $O M N e T++$ modules. An important group of functions is related to the centralized creation and destruction of processes with unique identification numbers. The hierarchy tree splits into common base classes for each general type of ProC/B-element. There are basic classes for sources, PCEs, FUs, sinks and connection elements. Figure 9 shows an extract of the inheritance hierarchy for all types of basic function units available in ProC/B's modeling library. The base class for function units named Ba seFU provides consistent handling of arriving and leaving processes including updates of statistic functions. For this reason, the handleMessage ( ) method required by $O M N e T++$ being implemented in every module is forked into specialized handlers inside the base classes. PCEs must implement handleProcessActivity () and FU handleProcessService () as shown later in Listings 3 and 4. Sources and sinks have similar constructs.

Assessing the way messages are handled in modules for $\operatorname{Pro} C / B$ elements also helps to realize timeouts for the activity 


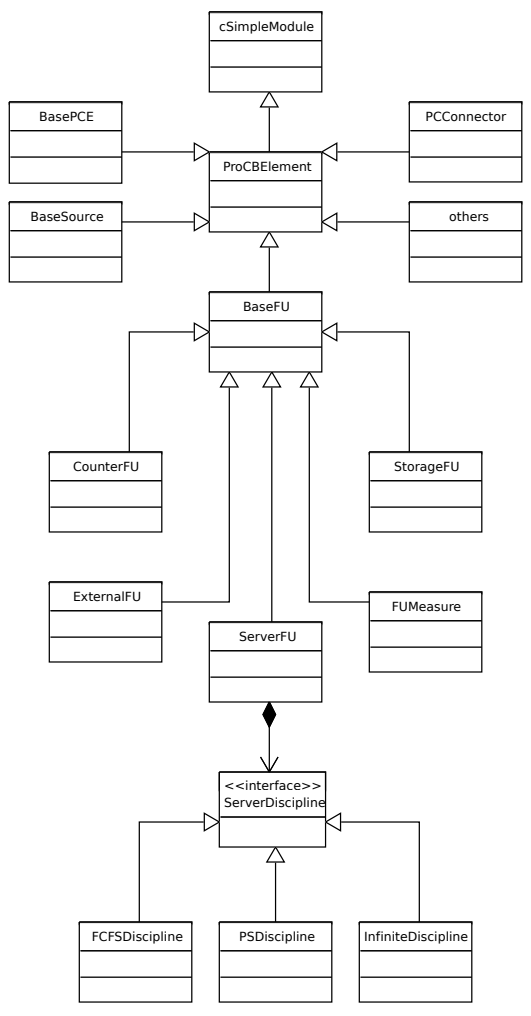

Figure 9: ProC/B's class hierarchy

duration of process chain elements. Each time a process chain element derived from type BasePCE performs a service call to a function unit, the process message is held back and replaced with a copy. The only difference between both messages is a new unique ID for the copy. Both ID numbers are stored in an associative collection. The copy will be sent to the called function unit while the original message still waits at the process chain element. When the service call is finished and the copy returns, the original message is deleted and the ID is transferred to the copy rendering it as the original message from now on. Recently we enhanced $P r o C / B$ by the option to set a timer before the copy is sent to the function unit (essentially this timer option makes copying of processes necessary). If the service call duration exceeds the time limit (i.e. the copy does not arrive in time) the original message is released and sent to the next process chain element. The ID of the copy is blacklisted and the copy is deleted when it arrives back later. Timeouts are a new feature realized in the ProC/B mapping to $O M N e T++$ and are not supported by the HIT simulation environment. An extensive description including examples can be found in [25].

Now we will describe two examples to explain our mapping of behavior more explicitly.

Figure 10 shows the symbol of a Delay PCE as a simple language element of $P r o C / B$. It has the task to delay arriving processes by some amount of time, either deterministic or by a random number from some predefined distribution. Listing 2 shows the corresponding *.ned-file used by $\mathrm{OMNeT++}$ to pass parameters to modules and define gateways where messages arrive and leave. 


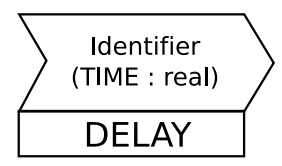

Figure 10: ProC/B's symbol for a delaying PCE

Listing 2: Source of DelayPCE.ned

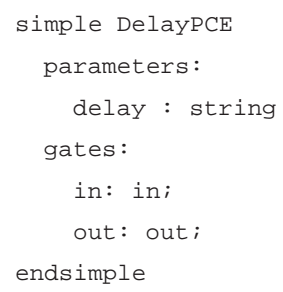

A process chain element DelayPCE is defined with just one pair of gates because it is connected to only two other process chain elements. Parameter delay is set by the *.ned-file instantiating this module as specified in the original ProC/B model. Implementations of DelayPCE subclassing CSimpleModule have to respect the fact that a second or third or an arbitrary number of processes can arrive while the first process is still delayed. The concrete implementation in Listing 3 is short, overloading the function handleProcessActivity (PCMessage* msg) and implementing the specific reaction on arriving messages. The delay time is generated from the predefined distribution, the following line in the code delays the incoming message by the amount of time using OMNeT++'s sendDelayed ( ) method. Please note that no messages are stored inside the module, allowing the module to accept an infinite number of processes. Method param () reassembles the well known method par () to fetch parameters stored in *.ned files. The new method parses expressions used in ProC/B models, which can be either arithmetic expressions or a ProC/B specific naming of random distributions.

All elements of ProC/B's process flow control had to be implemented as modules, too. As described before, process chain connectors (Fig. 11) are used to synchronize processes. Their implementation as OMNeT++ module is shown in Listing 4.

Process messages arrive at the input gates of the module. They are stored immediately in queues, one queue for each input gate. Pro $C / B$ allows us to specify a number of processes per gate that are required to start a transition which realizes the synchronization. Such semantics similar to token consumption in Petri Nets can for example be used to model usage of goods in production processes. The exact numbering is read from the process parameters of the arriving message in the first loop. If

Listing 3: Source of DelayPCE.cc

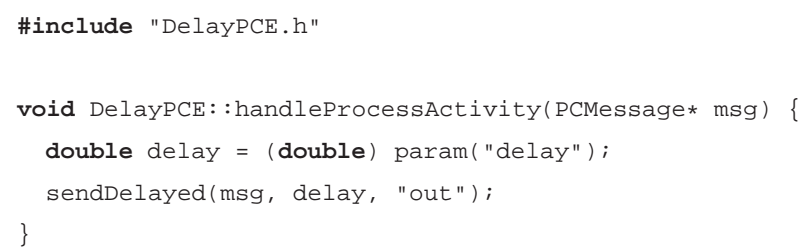




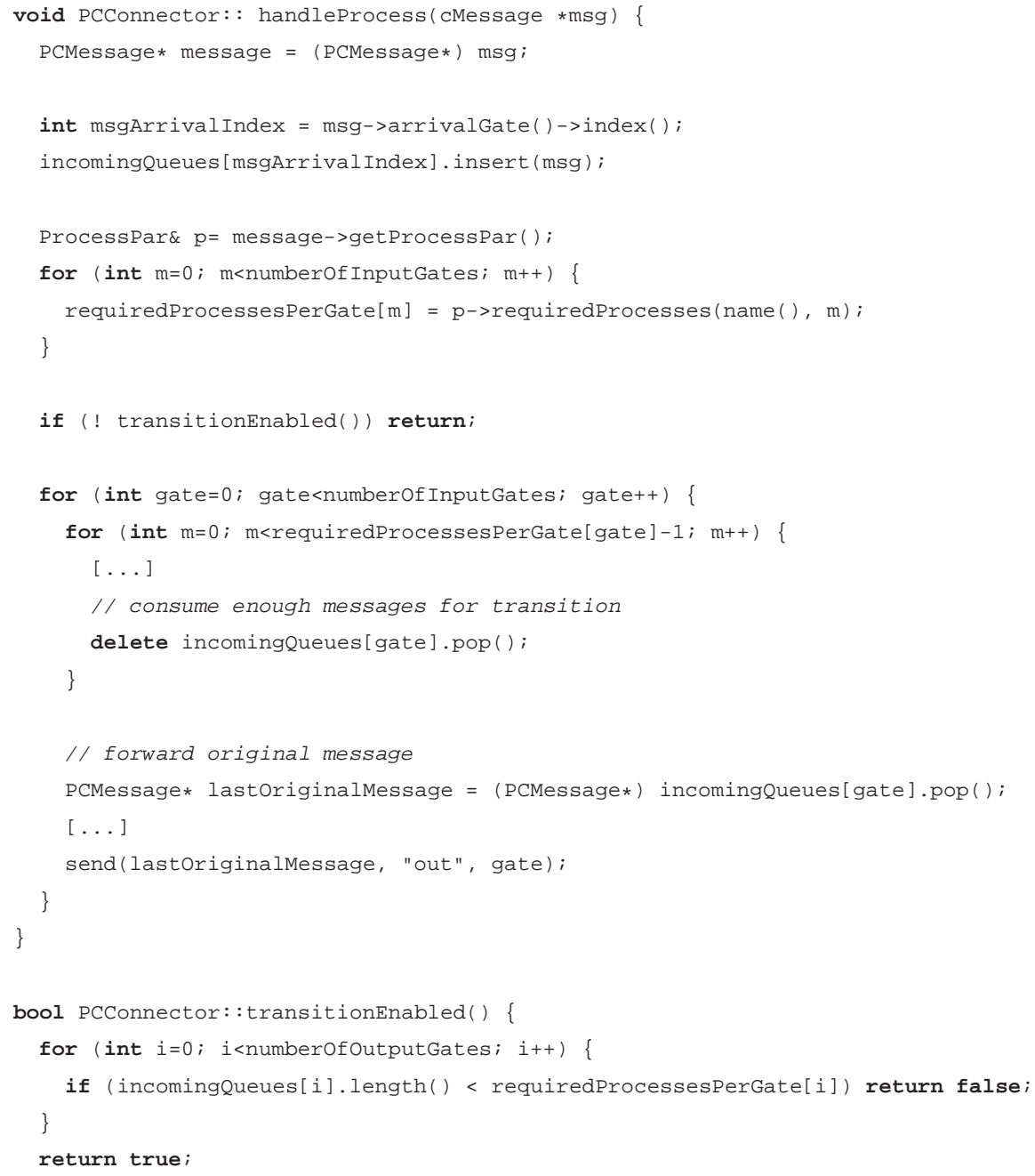




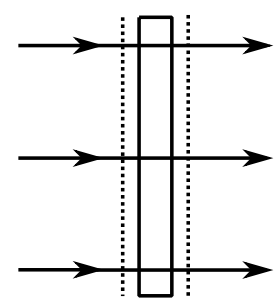

Figure 11: PCConnector in ProC/B for synchronization of three chains

a sufficient number of processes arrived (at least one per gate) the sentinel condition transitionEnabled () will allow the transition to fire. Otherwise the transition has to wait for additional processes. After synchronization all processes will arrive at the next element in their process chain at the same time.

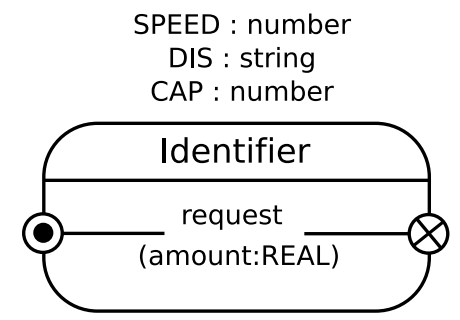

Figure 12: Server Function Unit in ProC/B

As an advanced example ServerFU is shown in Fig. 12. It represents a set of limited and identical resources which processes can request and use. ServerFU offers the service "request" to PCEs, a parameter for the requested amount of service has to be passed with the calling message.

Listing 5: ServerFU.ned

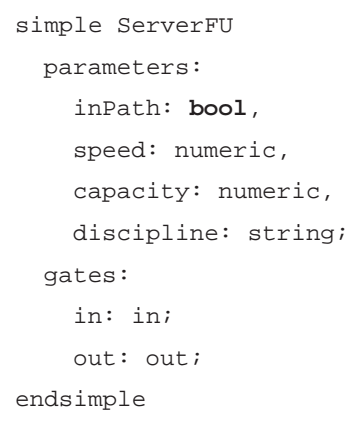

Listing 5 contains the definition of ServerFUs in $O M N e T++$ 's modeling language. Three parameters are passed to the module by $O M N e T++$ at runtime:

speed of a resource to execute service calls. This means that calling PCEs request an amount of service according to some average resource. The concrete FU can be faster ( speed $>1$ ), slower $($ speed $<1$ ) or an average resource $($ speed $=1)$. 
capacity number of resources offered by the server

discipline the resource scheduling the server uses.

By offering a single service, ServerFU only needs one pair of gates. As stated before, messages are delivered to these gates by direct send calls, making it unnecessary to connect this module with other elements in the model.

The parameter discipline plays an important role in ProC/B models, as the behavior of function units are matched to the way resources are shared in the real system. Currently three scheduling disciplines can be mapped from ProC/B to OMNeT++:

FCFS queues serve requests for resources by the rule "first come, first served". Processes which obtained a resource, allocate it according to the amount defined by the constant or distribution of the service call and parameter speed.

IS "Infinite Server", every request is immediately granted and takes the time specified by the service call and parameter speed.

PS "Processor Sharing", all requests are immediately granted. Every process makes use of the full set of resources (accelerating service time by the number of resources), but has to share resources with other processes using the server at the same time. Capacity is distributed uniformly among all processes (slowing down execution time by the reciprocal value of the number of processes).

It is possible to interpret these three types of scheduling as different kinds of servers, yet their implementation in $O M$ $\mathrm{NeT++}$ uses only one module to simplify the structure mapping by procb2ned. Internally ServerFU makes use of the strategy pattern [26] to vary it's behavior according to parameter discipline.

An excerpt of ServerFU's source is shown in Listing 6. The handleMessage ( ) method is divided into two parts by an if clause, newly arriving process messages with selfMessage set to false are served in the lower part.

At the beginning, welcomeMessage computes some basic statistics of arriving processes as described in section 4 and increments numberMsgInSystem. Additionally, the ServerFU pushes its name in writeNameInPath on the stack keeping track of every process chain element the message passed through. The next line is part of the strategy pattern, discipline holds objects of type ServerFU : Discipline encapsulating FCFS, Infinite Server or Processor Sharing as described above. Those strategies are instantiated depending on parameter discipline in Listing 5 on the module's initialization. Their behavior on newly arriving processes is specified in handleProcess ( ). Here we present the methods FCFS and IS as examples: In FCFS, time is granted to processes as long as the servers capacity is not exceeded. Otherwise the process is enqueued until resources become available. The Infinite Server is even more simple, it just accepts every process.

In both examples, time consumption is modeled by scheduling process messages to the function unit itself, adding the amount of time the service will take before sending. When the message returns, selfMessage is true and the upper part of handleMessage () is executed. Again, an object of type ServerFU: :Discipline handles processes a second time. In FCFS, the first process message waiting for free resources is removed from the queue and immediately scheduled for completion of the service. For Infinite Server no further action after service completion is necessary. 


\subsection{Translation of Result Measures}

The main focus when analyzing a simulation model is on determining quantitative results for the model, like for example throughputs or response times. ProC/B offers the possibility to measure properties at every FU, though depending on the type of the FU the available properties may differ: Throughput, response time and population can be measured at any FU. Additionally, for every server the utilization and for every storage the state can be examined. For composed FUs the modeler may define further measures (called rewards in ProC/B). ProC/B allows for three different types of rewards: count, event and state. Rewards of the type event can be used for serially collecting values, rewards of the type count for estimating rates and rewards of the type state for the description of trajectories. Those types are used for the realization of standard measures like throughput or response time as well. While the user-defined measures have to be updated manually (ProC/B provides a model element for updating those rewards), the standard measures are updated automatically whenever a process enters or leaves a FU.

As already mentioned $P r o C / B$ allows for streams to be itemized in detailed ways. This enables for example the measurement of the train population at the terminal in Fig. 2 without counting trucks. To achieve this, the modeler can specify a path consisting of elements in the ProC/B model. Only processes, that have moved through all of the specified elements will be considered when updating the stream. Most of the described features available in ProC/B are derived from the measures that $H I T$ offers, thus allowing an easy transformation from ProC/B to HIT.

Currently when analyzing the model with HIT, streams of data are generated during simulation, which are basically lists of pairs consisting of a time stamp and some associated value. This data is used to calculate the usual characteristics like mean, standard deviation and confidence intervals for the different measures. The ProC/B toolset contains a tool, that generates plots and visualizes the simulation results.

When using $O M N e T++$ for simulation the key features like itemizing streams as well as the output data of the simulation should be preserved, so that this new simulation environment fits into our existing toolset. While $O M N e T++$ offers basic facilities for measurement in e.g. communication protocols, it needs to be extended to meet the demands for the simulation of logistics networks.

In the remainder of this section it is shown how the measurement is implemented for Standard-FUs like servers or storages. Measuring properties at composed FUs requires some additional effort and is presented afterwards. Finally the itemizing of streams is explained.

For Standard-FUs the measurement streams have to be updated when a process enters (which means a service of the FU has been requested by a process) or leaves the FU. In the $O M N e T++$ representation of the model a service request is indicated by a message sent to the FU. The population is updated whenever a process enters or leaves the FU, throughput and response time are updated when a process leaves the FU. For Standard-FUs (like server or storage) the data collection and evaluation is implemented as $\mathrm{C}++-$ Code within the corresponding simple modules. This brings up problems for composed FUs: When the $P r o C / B$ model is translated to an $O M N e T++$ representation, composed FUs are represented as compound modules, thus only a *.neddescription exists that lacks the ability to implement code for measurements. Therefore the module of every composed FU contains a specific simple module called FUMeasures (see Fig. 13) to realize measurements in composed 
FUs.

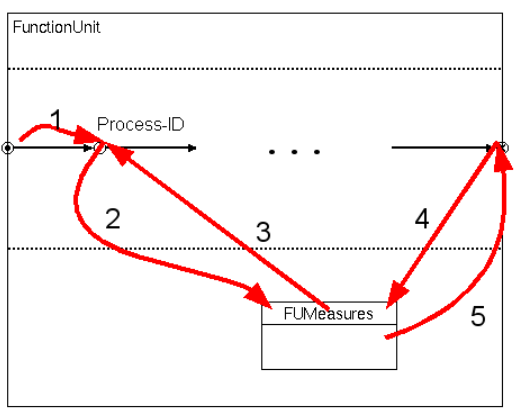

Figure 13: Message flow for the measurement in composed FUs

Fig. 13 shows the message flow that is necessary for measurements in composed FUs. Starting at the source a message is sent to the Process-ID (Process-IDs are used for the identification of a process chain and the declaration of local variables and are always connected with the source of a process chain). From there a message is sent to the special module FUMeasures. This module has no counterpart in $P r o C / B$ and its sole purpose is to enable the measurement in composed FUs. After the message is returned to the Process-ID, further elements of the process chain are processed (denoted by three dots in Fig. 13). When the process has reached the sink, the message is sent to the module FUMeasures and back to the sink again. All update operations of streams for a FU are performed within FUMeasures. The first message (sent by the Process-ID) means that a process has entered the FU (and thus the population is updated for example), the second message (sent by the sink) means that a process is leaving the FU again. Although FUMeasures is not an element of the ProC/B language it is part of the inheritance hierarchy of function units (cf. Fig. 9) in the Оsimu library. The superclass BaseFU adds the ability to return incoming process messages to the sending Process-ID or sink. Similar to calls of other FUs a stack of senders inside PCMessage is used to find the originator.

As already mentioned, $P r o C / B$ allows one to specify a path consisting of elements, so that only processes, that moved along that path through the model will be considered when updating measures. Those paths are part of the ProC/B experiment description and need to be translated to the $O M N e T++$ model and taken into account during the update of measurement streams. When mapping to $O M N e T++$ the ProC/B experiment description is saved in an *ini-file, that is loaded when the simulation starts. Additionally, a parameter is set for each element appearing in one of the paths in the corresponding *.ned-file when the model structure is mapped. During simulation the path a message takes through the model is saved and compared with the paths that have been specified in the ProC/B experiment. To store this information a new message class is used, that can carry the path information. Updates of the measurement streams are only performed when the path of the message matches one of those paths from the ProC/B experiment.

$O M N e T++$ provides several classes for the collection of data and the generation of statistical measures like mean or standard deviation which are derived from the abstract class CStat istic. Because the calculation of means does not match the specification of the streams in $\mathrm{Pro} C / B$, our ProC/B to $O M N e T++$ implementation provides its own classes (derived from CStatistic) to generate statistics (one for each of the stream types event, state and count mentioned before). For the 
estimation of confidence intervals the batch means-method [27] is used. The generated output is saved in the same format as the output of HIT, so that our existing tools can parse the data for result presentation.

\section{Validation of the Transformation}

If different tools are used to simulate a model, then it is necessary that the semantics of the model is the same in every simulation tool. Ideally, identity of semantics should be proved formally. A formal proof would require a formal semantics such that formal analysis techniques are applicable to check equivalence. Unfortunately, simulation models are much too complex to be described in simple languages that allow a formal analysis. This implies that identical behavior of models cannot be strictly verified, it can only be validated.

Pro $C / B$ was designed to introduce a well defined semantics and an automated analysis to hierarchical process chain models. A specification was laid down in [23], describing the semantics of PCEs and FUs in an operational form. Many aspects of the operational semantics are implicitly defined by the HIT runtime environment. Thus, the behavior of the HIT simulation model is the behavior which should be observed when $O M N e T++$ executes the model. However, the operational semantics depends on several aspects like execution order of simultaneous events, the order of initialization which are generally not well defined in discrete event simulation and, additionally, the realization of random processes that depend on the random number generator.

We distinguish between validation of models with and without random numbers. The former will be named deterministic models, although this is not strictly correct since simultaneous events may yield a non deterministic behavior. For deterministic models behavior can be compared using traces. Although, HIT and OMNeT++ both have a trace function it is not recommended to use these functions for comparisons since the format differs and cannot be easily transformed from one to another. Instead models are augmented with code PCEs including output statements. Such PCEs can be added to every PC. Thus, HIT and $O M N e T++$ generate the same trace output which can be easily compared.

To prove equality of traces we developed an automated testing environment to compare output of ProC/B models analyzed with $H I T$ and $O M N e T++$. It is based on a set of simple and deterministic ProC/B models, designed to test the behavior of exactly one element of ProC/B's language. Driven by our batch testing environment, identically formatted output of $H I T$ and $O M N e T++$ is compared by an awk script, highlighting differences in measurement results and event traces. Additionally, a selection of deterministic models taken from former projects is also subject to comparison, making sure that our ProC/B language elements implemented in modules interact correctly.

Testing nondeterministic models is limited since different random number generators are used in HIT (actually implementations of SIMULA) and OMNeT++. So, even starting with same seeds, results and event orders will differ. Consequently, we can only check in a statistical sense whether the implementation is correct, i.e., the different language elements behave identically. For this purpose, animations can be compared, traces can be visualized and results can be compared using statistical test. A typical approach is to estimate the same measure with both simulators, HIT and OMNeT++, and then statistically evaluate a random variable describing the difference between both measures. This can be done by comparing confidence intervals or using statistical tests (for details see e.g. [24][chap. 10]). 
Table 1 shows simulation results of an M/M/1 system $(\rho=0.5)$ as an example of a simple nondeterministic system included in our testing environment. Results are sufficiently close to assume an equivalent behavior for this model with a high significance probability. The second example are values taken from a central server system [28], also a nondeterministic system. Comparisons of simulation results in table 2 also indicate equivalence. A detailed analysis of the central server system with ProC/B is available in [29].

We additionally compared several simulation results of larger models and obtained similar minor differences (cf. Sect. 6).

Table 1: M/M/1 system simulation results (90\% confidence interval)

\begin{tabular}{|c|c|c|c|}
\hline & Population & Throughput & Response time \\
\hline \multirow{2}{*}{ HIT } & 1.00202 & 1.00023 & 1.001789 \\
& $\pm 0.18 \%$ & $\pm 0.05 \%$ & $\pm 0.16 \%$ \\
\hline \multirow{2}{*}{ OMNeT++ } & 1.00126 & 0.99964 & 1.000905 \\
& $\pm 0.33 \%$ & $\pm 0.14 \%$ & $\pm 0.22 \%$ \\
\hline
\end{tabular}

Table 2: Comparison of simulation results for Central Server (90\% confidence interval)

\begin{tabular}{|c|c|c|c|}
\hline & Population & Throughput & Response time \\
\hline HIT & 1.1559 & 0.7491 & 1.5429 \\
& $\pm 0.16 \%$ & $\pm 0.15 \%$ & $\pm 0.23 \%$ \\
\hline \multirow{2}{*}{ OMNeT++ } & 1.1548 & 0.7499 & 1.5398 \\
& $\pm 0.15 \%$ & $\pm 0.15 \%$ & $\pm 0.23 \%$ \\
\hline
\end{tabular}

\section{Comparison of performance and simulation results}

Though our implementation of ProC/B on $O M N e T++$ is not as mature as the one on HIT, we achieved promising runtime results. Times in table 3 were taken for analyzing a model over 1.000 .000 time units omitting model initialization and output.

Table 3: Runtime comparison

\begin{tabular}{|c|c|c|}
\hline Model & HIT & OMNeT++ \\
\hline $\mathrm{M} / \mathrm{M} / 1$ & 0 min. $57 \mathrm{sec}$. & 0 min. $22 \mathrm{sec}$. \\
\hline Central Server & 0 min. $35 \mathrm{sec}$. & 0 min. $24 \mathrm{sec}$. \\
\hline Freight Village & 8 min. $36 \mathrm{sec}$. & 2 min. $33 \mathrm{sec}$. \\
\hline
\end{tabular}


Our examples indicate that $O M N e T++$ is in most cases about 2 to 3 times faster than HIT.

The runtime comparison in table 3 features the $\mathrm{M} / \mathrm{M} / 1$ system mentioned before as well as the execution time of the freight village model introduced in Sect. 2.1. The measurements for the central server system show an improvement in runtime of only approx. $30 \%$ for $O M N e T++$. Since the model is based on a closed loop with only two active processes, less CPU time for process incarnations and queuing is used such that the difference between the two tools shrinks [29]. Still, a significant speed up can be noticed.

The values given for $O M N e T++$ are preliminary as we focussed on correct mapping of behavior and ignored performance issues for the time being. Performance bottlenecks still exist in statistical methods and dynamic search of matching function units to PCEs.

Table 4 shows some simulation results for the model of the freight village from Sect. 2.1. The table contains population, throughput and response time for the FU forklifts (see Figs. 2 and 3) estimated with HIT and OMNeT++. As one can see the results are similar.

Table 4: Comparison of simulation results for the server forklifts ( $95 \%$ confidence interval)

\begin{tabular}{|c|c|c|c|}
\hline & Population & Throughput & Response time \\
\hline HIT & $\begin{array}{c}4.988794 \\
\pm 0.30521 \%\end{array}$ & $\begin{array}{c}0.366475 \\
\pm 0.312747 \%\end{array}$ & $\begin{array}{c}13.612308 \\
\pm 0.633167 \%\end{array}$ \\
\hline OMNeT++ & 5.02047 & 0.36527 & 13.744485 \\
& $\pm 0.752181 \%$ & $\pm 0.353753 \%$ & $\pm 0.895352 \%$ \\
\hline
\end{tabular}

\section{Conclusions}

$O M N e T++$ is an environment which has been mainly designed for the simulation of communication networks. In this paper we demonstrated how to enable $O M N e T++$ for being used in other areas. We described the automated transformation of hierarchical process chains specified by $P r o C / B$ models to corresponding hierarchical $O M N e T++$ models.

Since the world views of $P r o C / B$ and $O M N e T++$ differ, the transformation is not straightforward and has to respect several special features of ProC/B. For example: Elements of the behavior description, like process chain elements (PCEs), are mapped to nodes, i.e. structural components, in the OMNeT++ description in order to exploit $O M N e T++$ 's animation capabilities. Furthermore additional elements for measurements are created as OMNeT++ elements which do not have a direct correspondence in the original ProC/B model.

The "correctness" of the transformation has been validated by several test models where we inserted special output commands, so that discrepancies from the execution via $O M N e T++$ and the reference simulator HIT can be detected automatically.

The current implementation is a prototype and future work will concentrate on further improvements of the simulation 
efficiency and the connection to existing ProC/B tools and $O M N e T++$ modeling features. One of the next steps will be the utilization of Akaroa parallel simulation libraries to reduce runtimes by using multiple computers in parallel. Furthermore we are continuing recent work [25] using the $O M N e T++$ framework INET for the combination of high-level service-oriented architecture (SOA) components and detailed lower level network architecture and protocols. In this approach SOA components and their orchestration are described in $P r o C / B$, and the network architecture is specified in $O M N e T++$. By means of the automated transformation of ProC/B models to $O M N e T++$ described in this paper, both models can be combined into a single executable simulation model of the overall system.

\section{References}

[1] D. W. Schunk and B. M. Plott. Using Simulation to Analyze Supply Chains. In Proceedings of the 32nd Winter Simulation Conference, pages 1095-1100, 2000.

[2] M. D. Rossetti and H.-T. Chan. A Prototype Object-Oriented Supply Chain Simulation Framework. In Chick et al. [30], pages $1612-1620$.

[3] ARIS business simulator, 2007. URL:http://www.ids-scheer.de/.

[4] J. Rathmell and D. T. Sturrock. The Arena Product Family: Enterprise Modeling Solutions. In Snowdon and Charnes [31], pages 165-172.

[5] M. W. Rohrer and I. McGregor. Simulating Reality Using AutoMod. In Snowdon and Charnes [31], pages 173-181.

[6] F. Bause, H. Beilner, M. Fischer, P. Kemper, and M. Völker. The ProC/B Toolset for the Modelling and Analysis of Process Chains. In T. Field, P. G. Harrison, J. T. Bradley, and U. Harder, editors, Computer Performance Evaluation / TOOLS, volume 2324 of Lecture Notes in Computer Science, pages 51-70. Springer, 2002.

[7] H. Beilner, J. Mäter, and N. Weißenberg. Towards a performance modelling environment: News on HIT. In R. Puigjaner and D. Potier, editors, Modeling techniques and tools for computer performance evaluation, pages 57-75, 1989.

[8] P. Buchholz, D. Müller, P. Kemper, and A. Thümmler. OPEDo: A Tool Framework for Modeling and Optimization of Stochastic Models. In L. Lenzini and R. L. Cruz, editors, Proceedings of the 1st International Conference on Performance Evaluation Methodologies and Tools, VALUETOOLS 2006, page 61. ACM, 2006.

[9] P. Kemper and C. Tepper. Traviando - Debugging Simulation Traces with Message Sequence Charts. In International Conference on Quantitative Evaluation of SysTems (QEST), pages 135-136. IEEE Computer Society, 2006.

[10] F. Bause, P. Buchholz, J. Kriege, and S. Vastag. Simulating Process Chain Models with OMNeT++. In Proc. of the First International Conference on Simulation Tools and Techniques for Communications, Networks and Systems (SIMUTools 2008), Marseille (France), March 2008.

[11] Omnet++ community side. URL:http//www.omnetpp.org/.

[12] J. Huang. Simulative Bewertung von ProC/B-Modellen. Master's thesis, Universität Dortmund, Fachbereich Informatik, Lehrstuhl 4, Dortmund, 2006.

[13] Q. Zhu. Beschreibung von ProC/B-Modellen zur simulativen Bewertung. Master's thesis, Universität Dortmund, Fachbereich Informatik, Lehrstuhl 4, Dortmund, 2006.

[14] Collaborative Research Center 559 “Modelling of Large Logistics Networks". http://www.sfb559.uni-dortmund.de.

[15] P. Buchholz and U. Clausen. Große Netze der Logistik - Die Ergebnisse des Sonderforschungsbereichs 559. Springer, 2009.

[16] M. B. Juric. Business Process Execution Language for Web Services. Packt Publishing Limited, 2006. 
[17] M. Arns and T. Härtel. BPEL für Prozessketten. In P. Buchholz, editor, Workshop ”Modellierung großer Netze in der Logistik”, Dortmund, Research Report 819/2008, Computer Science, TU Dortmund, 2008.

[18] Osman Balci. Verification, Validation, and Certification of Modeling and Simulation Applications. In Chick et al. [30], pages $150-158$.

[19] T. Murata. Petri Nets: Properties, Analysis and Applications. Proc. of the IEEE, 77:541-580, 1989.

[20] F. Bause and J. Kriege. Detecting Non-Ergodic Simulation Models of Logistics Networks. In Proc. of the Second International Conference on Performance Evaluation Methodologies and Tools (VALUETOOLS 2007), October 2007.

[21] P. Buchholz and C. Tepper. Functional Analysis of Process Oriented Systems. In H. Fleuren, D. den Hertog, and P. Kort, editors, Operations Research Proceedings, pages 127-135. Springer, 2005.

[22] H. Beilner, J. Mäter, and C. Wysocki. The Hierarchical Evaluation Tool HIT. In Short Papers and Tool Descriptions of the 7th International Conference on Modelling Techniques and Tools for Computer Performance Evaluation, 1994.

[23] F. Bause, H. Beilner, and M. Schwenke. Semantik des ProC/B-Paradigmas. Technical Report 03001, ISSN 1612-1376, Sonderforschungsbereich 559 "Modellierung großer Netze in der Logistik", 2003.

[24] W. D. Kelton and A. Law. Simulation Modeling and Analysis. McGraw Hill, 2000.

[25] F. Bause, P. Buchholz, J. Kriege, and S. Vastag. A Framework for Simulation Models of Service-Oriented Architectures. In S. Kounev, I. Gorton, and K. Sachs, editors, SPEC International Performance Evaluation Workshop 2008 (SIPEW 2008), pages 208-227. LNCS 5119, Springer, June 2008.

[26] E. Gamma, R. Helm, R. Johnson, and J. Vlissides. Design Patterns. Elements of Reusable Object-Oriented Software. Addison-Wesley, 1995.

[27] G.S. Fishman. Discrete-Event Simulation Modeling, Programming and Analysis. Springer, 2001.

[28] S. Lavenberg. Computer Performance Modeling Handbook. Academic Press, 1983.

[29] Jan Kriege and Sebastian Vastag. ProC/B goes OMNeT++: Efficient Simulation of Process Chains. In Falko Bause and Peter Buchholz, editors, Proc. of the 14th GI/ITG Conference on Measurement, Modeling, and Evaluation of Computer and Communication Systems (MMB 2008), pages 303-305, Dortmund, 2008. VDE Verlag.

[30] S. E. Chick, P. J. Sanchez, D. M. Ferrin, and D. J. Morrice, editors. Proceedings of the 35th Winter Simulation Conference: Driving Innovation, New Orleans, Louisiana, USA, December 7-10, 2003. ACM, 2003.

[31] J. L. Snowdon and J. M. Charnes, editors. Proceedings of the 34th Winter Simulation Conference: Exploring New Frontiers, San Diego, California, USA, December 8-11, 2002. ACM, 2002. 


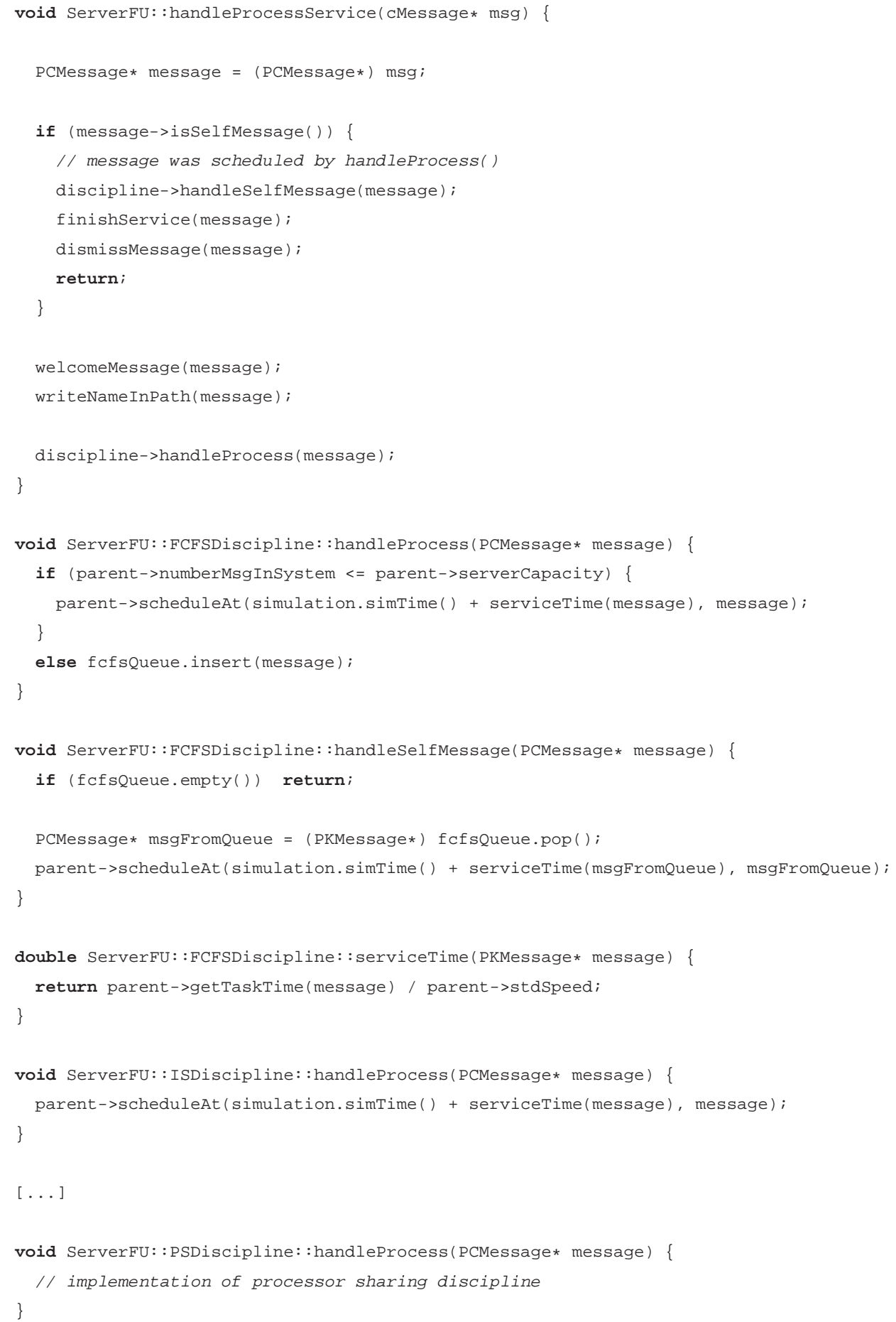

